\title{
The developmentally-timed decay of an essential microRNA family is seed sequence-dependent
}

Bridget Donnelly ${ }^{1,2}$, Bing Yang ${ }^{1}$, Chen-Yu Liư ${ }^{1}$, Katherine McJunkin ${ }^{1,3}$

$5 \quad{ }^{1}$ Laboratory of Cellular and Developmental Biology, NIDDK Intramural Research Program, 50 South Drive, Bethesda, MD, 20815, USA

2Johns Hopkins University Department of Biology, 3400 N. Charles Street, Baltimore, MD, 21218, USA

10

${ }^{3}$ Correspondence: mcjunkin@nih.gov

\section{Abstract}

MicroRNA (miRNA) abundance is tightly controlled by regulation of biogenesis and decay. Here we show that the mir-35 miRNA family undergoes regulated decay at the transition from embryonic to larval development in $C$. elegans. The seed sequence of the miRNA is necessary and sufficient for this regulation. Sequences outside the seed (3' end) regulate mir-35 abundance in the embryo but are not necessary for sharp decay at the transition to larval development. Enzymatic modifications of the miRNA 3' end are neither prevalent nor correlated with changes in decay, suggesting that miRNA 3' end display is not a core feature of this mechanism and further supporting a seed-driven decay model. Our findings demonstrate that seed sequence-specific decay can selectively and coherently regulate all redundant members of a miRNA seed family, a class of mechanism that has great biological and therapeutic potential for dynamic regulation of a miRNA family's target repertoire.

\section{Introduction}

microRNAs (miRNAs) are small non-coding RNAs ( 22-23 nucleotides) that, when bound by Argonaute, form the miRNA-induced silencing complex (miRISC) and negatively regulate target mRNAs (Dallaire et al., 2018). The biogenesis of miRNAs has been well described; first, miRNAs are transcribed as a primary miRNA (pri-miRNA): a long transcript containing a $\sim 35$ base

30 pair stem-loop structure formed by intramolecular base-pairing (Fang and Bartel, 2015; Han et al., 2006; Ma et al., 2013; Zeng et al., 2005). The double-stranded RNA hairpin structure of the pri-miRNA is recognized by the Microprocessor complex (Drosha and DGCR8/Pasha) (Fang and Bartel, 2015; Han et al., 2006; Ma et al., 2013; Zeng et al., 2005). The catalytic RNase III domain of Drosha cleaves the pri-miRNA, resulting in the generation of a miRNA precursor (pre-miRNA) hairpin structure with a two-nucleotide overhang at the 3' end (Denli et al., 2004; Gregory et al., 
2004; Han et al., 2004; Landthaler et al., 2004). Once exported from the nucleus, the pre-miRNA is cleaved by the RNase III enzyme Dicer into a $\sim 22-23$ nucleotide duplex that is loaded into Argonaute (Bernstein et al., 2001; Grishok et al., 2001; Hutvágner et al., 2001; Ketting et al., 2001; Knight and Bass, 2001). The mature guide strand remains in the Argonaute protein, becoming a part of the miRISC, while the star strand is ejected and degraded (Iwasaki et al., 2010, 2015). Once the mature miRNA is incorporated into miRISC, miRISC uses the bound miRNA guide strand to target complementary regions in the 3'UTR of mRNAs to silence gene expression by translational repression, deadenylation and decay of the target mRNA (Dexheimer and Cochella, 2020).

The interaction between the miRNA and mRNA target is primarily mediated through nucleotides 2-8 at the 5' end of the miRNA (Brennecke et al., 2005; Lewis et al., 2003). This region, called the seed sequence, is the defining characteristic of a miRNA family, a group of miRNAs that act largely redundantly on an overlapping set of target genes due to their identical seed sequences (Alvarez-Saavedra and Horvitz, 2010; Parchem et al., 2015). Additional 50 supplemental base pairing between the 3' end of the miRNA and the target RNA occurs in some cases, conferring some differences in target repertoire of miRNA family members (which share a seed sequence but may differ in their 3' sequences) (Broughton et al., 2016; Brancati and Grosshans, 2018; Helwak et al., 2013; Ye Duan, Isana Veksler-Lublinsky, 2021).

While much is known about the biogenesis and functions of miRNAs, relatively little is known about the mechanisms of decay of mature miRNAs. While half-lives of miRNAs vary, what determines these differences in stability is for the most part unknown (Bail et al., 2010; Kingston and Bartel, 2019; Lehrbach et al., 2012; Marzi et al., 2016; Miki et al., 2014; Reichholf et al., 2019; Vieux et al., 2021). Thus far, multiple phenomena regulating miRNA stability have been observed, with different degrees of sequence-specificity.

Some decay pathways appear to be largely independent of miRNA sequence. In $C$. elegans, the 5' to 3' nuclease XRN-2, along with DCS-1, maintain wild type miRNA levels by degrading many (though not all) miRNAs (Bossé et al., 2013; Chatterjee et al., 2009). In the mouse retina, an undefined mechanism induces decay of most miRNAs upon light-dependent neuronal activity (Krol et al., 2010). At the maternal to zygotic transition in Drosophila, terminal 65 adenylation of maternal miRNAs by the noncanonical poly(A) polymerase, Wispy, induces their wholesale clearance (Lee et al., 2014). In other species, 3' nucleotide addition (tailing) has also been proposed to destabilize miRNAs in a sequence-independent manner (Boele et al., 2014; 
Katoh et al., 2015; Knouf et al., 2013; Lee et al., 2019; Shukla et al., 2019; Wyman et al., 2011; Yang et al., 2020a).

Other miRNA decay pathways are guided by moderate sequence-specificity. One example is Tudor SN-mediated miRNA decay (TumiD) (Elbarbary et al., 2017a, 2017b). In TumiD, the endonuclease Tudor-SN (TSN) cleaves a few dozen miRNAs at CA and UA dinucleotides greater than 5 nucleotides away from the 3' and 5' ends of the miRNA (Elbarbary et al., 2017a, 2017b). A more specific phenomenon confers instability to several members of the extended miR-

7516 family; this decay is dependent on sequences in the both the seed and the 3' portion of the miRNA (Rissland et al., 2011). Rapid decay of miR-382 is dependent on the 7 nucleotides at the 3' end of the miRNA (Bail et al., 2010).

The most sequence-specific mechanism of miRNA decay is called target-directed miRNA degradation (TDMD). TDMD occurs when a high-abundance RNA (the TDMD "trigger") binds to

80 a miRNA with extensive complementarity to both the seed sequence and the 3' half of the miRNA (Ameres et al., 2010; Baccarini et al., 2011; Bitetti et al., 2018; Cazalla et al., 2010; Ghini et al., 2018; Kleaveland et al., 2018; Libri et al., 2012; Marcinowski et al., 2012; la Mata et al., 2015; Piwecka et al., 2017). This extensive base pairing induces a conformational change that pulls the 3' end of the miRNA out of the PAZ domain of Argonaute, making it accessible to modification by untemplated nucleotide additions (tailing) and exonucleolytic cleavage (trimming) (SheuGruttadauria et al., 2019; Yang et al., 2020a). Recently, the Cullin-RING E3 ubiquitin ligase ZSWIM8 was identified as an effector of TDMD, leading to the model that the Argonaute conformation induced by extensive base pairing is recognized by ZSWIM8 for ubiquitylation and subsequent decay of the miRNA:Argonaute complex (Han et al., 2020; Shi et al., 2020).

The regulation of miRNA expression during development is crucial to ensure properly timed developmental transitions, but the extent to which miRNA decay contributes to ensuring proper temporal expression patterns of miRNAs and how development is coupled to regulated decay are not known.

In this work, we set out the examine the mechanism of decay of the mir-35 family of 95 miRNAs. The mir-35 family consists of 8 miRNAs, mir-35-42 (Alvarez-Saavedra and Horvitz, 2010). The mir-35 family members are maternally contributed as well as zygotically expressed in early embryogenesis, and they are sharply degraded at the transition from embryo to the first larval stage, L1 (Stoeckius et al., 2009; Wu et al., 2010). Understanding the mechanism of this decay will shed light on how selective miRNA decay occurs and how it is coupled to development. 
The mir-35 family is one of two miRNA seed families that are necessary for $C$. elegans embryogenesis. Because of their identical seed sequences, the mir-35 family members are functionally redundant; deletion of any single miRNA has no phenotypic consequences, whereas deletion of the whole family results in embryonic lethality (Alvarez-Saavedra and Horvitz, 2010). The mir-35 family miRNAs also play multiple roles in development, including promoting maximal 105 fecundity, ensuring sex determination, and regulating cell death (Doll et al., 2019; Kagias and Pocock, 2015; Liu et al., 2011; Massirer et al., 2012; McJunkin and Ambros, 2014, 2017; Sherrard et al., 2017; Tran et al., 2019; Yang et al., 2020b; Zhao et al., 2019).

How the mir-35 family is targeted for selective decay at the end of embryogenesis is not known. A recent study showed that the TDMD factor ZSWIM8 (known as EBAX-1 in C. elegans) 110 drives instability of the mir-35 family, suggesting that the mir-35 family is subject to TDMD (Shi et al., 2020; Wang et al., 2013). However, positions of the miRNA that are usually involved in the base-pairing interactions that drive TDMD (in the 3' half of the miRNA) are highly degenerate across the mir-35 family members (Figure 1A), suggesting that the mechanism of mir-35 decay may differ from previously-described examples of TDMD, and may represent a novel type of 115 selective miRNA decay mechanism.

Here we show that the mir-35 family is regulated at the level of decay at the embryo to L1 transition in C. elegans. We demonstrate that the seed sequence of mir-35 is necessary and largely sufficient for this developmentally timed decay. This decay is not correlated with high levels of miRNA 3' tailing and trimming. Together, these data suggest that this miRNA family is regulated

120 by a distinct - but possibly related - mechanism to TDMD. Seed-specific decay mechanisms such as this are likely to be more widespread in biological systems since they have potential to coregulate all members of a redundant miRNA family, potentially allowing dynamic derepression of the miRNA family's target genes.

\section{Results}

\section{mir-35 decay is seed sequence-dependent}

The mir-35 family is selectively decayed at the embryo to L1 transition (Stoeckius et al., 2009; Wu et al., 2010). We wondered if its decay is a regulated process or, alternatively, just a result of transcriptional shutoff in late embryogenesis. We also wondered whether its 130 characteristic seed sequence played a role in this putative regulated decay. To this end, we used CRISPR/Cas9 to mutate the genomic locus which encodes seven of the eight mir-35 family 
members, mir-35-41, on a single transcript (Figure 1B). (mir-42 is clustered with unrelated miRNAs at a different genomic locus.) We made targeted mutations to the seed sequence of the first hairpin in the mir-35-41 cluster (mir-35) using CRISPR/Cas9. This approach leaves the 135 remainder of the mir-35-41 cluster intact, which serves two purposes: 1) mir-35 loss-of-function phenotypes are not induced since the other family members remain wild type, and 2) mir-36-41 serve as internal controls derived from the same transcript as mir-35. Both strands of the hairpin encoding mir-35 were mutated to preserve secondary structure and support efficient processing (Figure 1B). One of the mutations was a reversal of the seed sequence, referred to hereafter as mir-35(seed_rev)), whereas the other mutation replaced the mir-35 seed sequence with random nucleotides (mir-35(seed_mut)) (Figure 1B).

To determine if biogenesis of mir-35 was affected by these seed mutations, we quantified mir-35 and the mutant variants using miRNA-Taqman qPCR, along with synthetic RNAs oligonucleotides to generate standard curves for absolute quantification (Figure S1). The embryo 145 concentration of mir-35(seed_ rev) is similar to wild type mir-35 (0.7-fold change), while mir35(seed_mut) is ten-fold lower (Figure 1C). To determine if the changes in the level of the mir-35 variants was at the level of biogenesis or post-biogenesis, we examined the abundance of their star strands in the embryo. Changes is abundance of the mir-35 variant star strands is similar to those in the respective guide strands (Figure 1D); these coupled changes suggest that the 150 decreased abundance of mir-35(seed_mut) is due to loss of efficiency in biogenesis.

Next, we examined whether the decay of mir-35 at the embryo to L 1 transition is altered by seed mutations. (Note that, because we use arrested L1 samples, post-embryogenesis growth has not begun, so any decreases in miRNA abundance must be attributed to decay rather than dilution caused by growth.) As expected, we observed a strong reduction in wild type mir-35 at 155 the transition from embryo to L1, with 12-fold lower abundance in L1 (Figure 1C). However, the decay of mutant mir-35(seed_rev) and mir-35(seed_mut) at the transition from embryo to L1 was greatly attenuated to essentially no change and 1.3-fold lower in L1 than embryo, respectively (Figure 1C). mir-35(seed_rev) derived from a second CRISPR allele with altered precursor secondary structure also showed attenuated decay (Figure S2). Therefore, the decay of mir-35 160 depends on its seed sequence. Importantly, the decay of mir-36 was not altered by the mutations in mir-35 (Figure 1C). This decoupling of the behavior of mir-35 and mir-36 - which share a primary transcript - further shows that mir-35 decay is regulated post-transcriptionally.

To confirm and extend these findings, we performed deep sequencing to profile all miRNAs. Deep sequencing confirmed that mir-35 was the only miRNA altered in abundance by 
165 these mutations in embryo or L1 samples (Figure S3). Consistent with the qPCR, wild type mir35 displayed sharp decay at the embryo to L1 transition, and the mir-35 seed mutants were resistant to this decay (Figure 1E). The decay of the other members of the mir-35 family was not affected by mir-35 seed mutations, despite most members sharing the mir-35-41 primary transcript (Figure 1E). Global analysis further demonstrated the selectivity of the decay of the mir-

17035 family in this developmental window: mir-35-41 represent seven of the eight most sharply downregulated miRNAs in this time point in wild type (Figure 1F). This analysis also reiterates the specificity of the effect of the mir-35 seed mutations, further demonstrating that the regulation of other miRNAs is not affected by these mutations (Figure 1F).

Together these results show that the decay of the mir-35 family at this developmental 175 transition is a regulated decay process (rather than the result of synchronous decay after transcriptional shutoff), since the behavior of miRNAs derived from the same transcript can be de-coupled. Furthermore, these results show that that the mir-35 seed sequence is required for this regulated decay.

mir-35 3' end mutants undergo efficient decay at the embryo to L1 transition

The necessity of the seed sequence for mir-35 decay (Figure 1) and the recent implication of the TDMD factor ZSWIM8/EBAX-1 in regulating stability of the mir-35 family (Shi et al., 2020) together suggest a TDMD-like decay mechanism. However, the degeneracy of sequences in the 3' region of the miRNA across the mir-35 family members (Figure $1 \mathrm{~A}$ ) suggests that the 185 mechanism may differ from previous examples of TDMD since multiple trigger RNAs would be necessary to bind with extensive complementarity to all the family members. (Note that results from mir-35(seed_rev) and mir-35(seed_mut) rule out an antisense RNA from the mir-35-41 cluster acting as a TDMD trigger RNA, since mutations at the genomic locus would not disrupt base-pairing with an antisense transcript.)

Therefore, we next investigated whether the 3' portion of the miRNA plays a role in mir-35 family decay and if the mir-35 seed sequence is sufficient for decay. To test this, we used CRISPR to generate two mir-35 mutant strains in which the non-seed ( 3 ' end) residues of the miRNA are mutated. The first mutant is comprised of the mir-35 seed sequence with a 3 ' end containing nucleotides that are either not present or rare among all mir-35 family members at a given 195 position, while preserving overall GC content (mir-35(mut_3')) (Figure 2A). The second mutant is a mir-35/mir-82 hybrid composed of the mir-35 seed sequence and mir-82 3' end (mir-35(mir- 
82_3')) (Figure 2A). The mir-82 sequence was chosen for the non-seed region of mir-35 because mir-82 expression is steady rather than downregulated at the embryo to L1 transition (Kato et al., 2009).

Again, we performed miRNA-Taqman qPCR of mir-35 and its mutant variants, using synthetic miRNAs of known concentrations to generate standard curves for absolute quantification (Figure S1). In embryos, the quantity of mir-35(mut_3') and mir-35(mir-82_3') were increased 20 -fold and 139 -fold relative to wild type mir-35, respectively (Figure 2B). To determine whether these changes in abundance arise during or after biogenesis, we also performed 205 absolute quantification of the star strands of the mir-35 variants. This analysis showed that the star strand abundances did not mirror changes in the guide strand abundances, suggesting that the large changes in guide strand abundance are post-biogenesis level effects, such as at the level of decay (Figure 2C). We therefore postulate that a second decay mechanism may act to limit abundance of mir-35 in the embryo in a manner dependent upon the 3' end sequence (Figure 210 S4).

We next measured the decay of the mir-35 3' end variants at the embryo to L1 transition. Unlike the seed mutants, the change in the abundance of the mir-35 3' end mutants from the embryo to L1 stage was similar to that of wild type mir-35 [7-fold for the mir-35(mut_3'), 14-fold for mir-35(mir-82_3'), and 8-fold for wild type] (Figure 2B). Likewise mir-36 was not affected by

215 the mutations (Figure 2B). Again, we confirmed and extended these findings using deep sequencing. Deep sequencing confirmed that the 3' end variants showed a similar depletion from embryo to L1 stage as wild type mir-35, and that no other miRNAs in the mir-35 family or otherwise were affected (Figure 2D-E, Figure S5). Thus, the sequence of the 3 ' end of the miRNA outside the seed did not affect the decay at this developmental transition.

Overall, we observed that seed mutations do not generally impact embryonic mir-35 abundance but strongly inhibit its decay at the embryo to L1 transition, whereas 3' end mutations strongly impact embryonic abundance of mir-35 but do not affect its decay at the embryo to L1 transition. Taken together, we propose that two decay mechanisms regulate mir-35 abundance: a 3' end-dependent mechanism limits abundance in embryos, while a seed-dependent 225 mechanism drives decay at the transition to L1 (Figure S4). Given that all positions 3' of the seed sequence are mutated in the 3' end mutants, the seed sequence of mir-35 is not only necessary but also largely sufficient to drive its regulated decay at the embryo to L 1 transition. 


\section{mir-35 variants are tailed and trimmed similarly to wild type mir-35}

While the seed-dependence and ZSWIM8/EBAX-1-dependence of mir-35 regulation suggest a TDMD-like mechanism, the dispensability of the 3' end for developmentally timed turnover suggests an alternative mechanism. Another hallmark of TDMD is a high prevalence of tailing and trimming during the decay process, which is thought to be due to conformational changes induced by extensive base pairing, that expose the 3' end of the miRNA to exonucleases

235 and nucleotidyltransferases.

To determine whether the mir-35 family bears this hallmark of TDMD, we examined the prevalence of tailing and trimming. We first examined the level of background in tailing measurements in our experimental and computational pipeline. To this end, synthetic miRNAs were spiked into total RNA after purification, and the amount of tailing called on these miRNAs is

240 considered background since these miRNAs were never present in the context of cellular lysate, so any apparent "tailing" must derive from errors introduced in cloning or sequencing. Tailing was below $1.5 \%$ in 317 out of 324 (98\%) such spike-in measurements, so tailing below $1.5 \%$ is considered background in these datasets. This threshold is marked by a dashed line on all tailing plots.

In embryos and L1s, we observed that miRNAs are tailed to various extents, though generally not very high levels (Figure 3A, Figure S6A). Tailing was mostly mono-uridylation, with some miRNAs displaying significant adenylation or cytidylation, as previously observed (Figure 3A, Figure S6A) (Vieux et al., 2021). Overall tailing and miRNA abundance were not correlated, and the mir-35 family members were generally high in abundance, with a wide range of tailing 250 frequencies observed across different members (Figure 3B).

We and others previously observed slight increases in the prevalence of tailed and trimmed miRNAs as miRNAs approach decay (Baccarini et al., 2011; Kingston and Bartel, 2019; Vieux et al., 2021). In TDMD, miRNAs experience very high levels of tailing and/or trimming (generally $\geq 20-40 \%$ tailed or trimmed isoforms) (Ameres et al., 2010; Baccarini et al., 2011; Bitetti

255 et al., 2018; Cazalla et al., 2010; Ghini et al., 2018; Kleaveland et al., 2018; Marcinowski et al., 2012). We hypothesized that the prevalence of tailed isoforms might increase at the embryo to L1 transition as the mir-35 family members undergo decay. Small increases in miRNA tailing and trimming were observed, but in most cases, these were not statistically significant, and the prevalence of modified isoforms remained modest (Figure 3C, 3E). 
We next examined the prevalence of tailed isoforms in the context of mutant versions of mir-35. Significant changes in tailing were observed, but interestingly, these did not correlate with changes in rates of decay (Figure 3D). For instance, mir-35(mut_3') was significantly more cytidylated and uridylated than wild type mir-35 in embryo and L1, and mir-35(mir-82_3') was significantly more adenylated, cytidylated, and uridylated than wild type mir-35 in both stages ( $p$ value $<0.05$ for all aforementioned comparisons) (Figure 3D). However, these two mir-35 variants displayed decay similar to that of wild type mir-35 at the embryo to L1 transition (Figure 2B). In contrast, mir-35(seed_rev) and mir-35(seed_mut) show similar amounts of tailing to the wild type mir-35 (Figure 3D), despite these variants' dramatically altered decay at the embryo to L1 transition (Figure 1C). Thus, changes in tailing did not correlate with changes in decay.

We next examined trimming of mir-35 variants. Like tailing, trimming varied widely among mir-35 variants, but not in a manner that correlated with the rate of decay. For instance, trimming increased most for mir-35(seed_mut), despite the enhanced stability of this variant (Figure 3F, Figure S6B). In contrast, mir-35(seed_rev) - which shows similarly enhanced stability - had no significant change in trimming (Figure 3F, Figure S6B). This isoform analysis also showed that

275 mir-35(mut_3') yields two major isoforms from biogenesis, the canonical 22-nt isoform and a 23nt isoform which is extended by $1 \mathrm{nt}$ at the $3^{\prime}$ end (Figure 3F, Figure S6B). Analysis of deep sequencing data showed that both isoforms are decayed similarly at the embryo to L1 transition (Figure S7). Overall, changes in trimming did not correlate with changes in decay.

All together, these data show that the tailing and trimming of the mir-35 family are much lower in prevalence than in known instances of TDMD, and that the incidence of trimmed and tailed isoforms across mir-35 variants did not correlate with rate of decay at the embryo to L1 transition. Together with the dispensability of the 3' end sequences of mir-35 for decay, this suggests that the mechanism of decay of mir-35 differs from previously described examples of TDMD.

\section{Reintroducing miRNA-target interactions does not restore decay of seed mutant variants} of mir-35

To further investigate the mechanism of mir-35 family decay at the embryo to L1 transition, 290 we examined the possible involvement of complementary RNA molecules as in TDMD. Decay of mir-35 at the embryo to L1 transition is dependent on its seed sequence but not its 3 ' end sequences, and canonical targets were previously shown to regulate miRNA stability in $C$. 
elegans (Chatterjee et al., 2011). We therefore wondered whether canonical miRNA:target interactions (rather than TDMD-like base-pairing) might play a role in mediating this decay. To

295 test this hypothesis, we restored canonical target interactions for a seed mutant variant of mir-35 to determine whether this restored developmentally-timed decay.

To this end, we sought to alter a similar stoichiometric proportion of the pool of mir-35 family miRNAs and the pool of mir-35 family targets. mir-35 makes up 20\% of the mir-35-42

300 miRNA molecules in embryos based on quantitative deep sequencing (Dexheimer et al., 2020), so we selected three target genes that together also make up $20 \%$ of the target molecules (as estimated from embryo RNAseq datasets) (Grün et al., 2014). These genes - egl-1, nhl-2, and sup-26 - were also selected because they are all validated targets that influence physiology downstream of mir-35-42 (Kagias and Pocock, 2015; McJunkin and Ambros, 2017; Sherrard et

305 al., 2017; Tran et al., 2019; Wu et al., 2010; Yang et al., 2020b). Using CRISPR, we made mutations to the mir-35 family binding site in the 3'UTR of these three target genes. These mutations enable binding by mir-35(seed_rev) rather than wild type mir-35, and we have previously shown that mir-35(seed_rev) represses such targets (Figure 4A) (Yang et al., 2020b).

We performed qPCR to measure the miRNA levels in the embryo and L1 stages. Again, we observed a significant reduction in wild type mir-35 from embryo to L1 and attenuated decay of the mir-35(seed_rev) (Figure 4B). Wild type mir-35 decay was not affected by the mutations of the target sites (Figure 4B). When mir-35(seed_rev) was combined with the mutant targets containing complementary binding sites, decay was similar to mir-35(seed_rev) without 315 engineered target interactions (Figure 4B). Thus, restoring interactions with canonical target genes is not sufficient to restore turnover of the mir-35 seed mutant.

\section{Discussion}

Here we investigate the regulation of the embryonically-expressed mir-35 family during 320 development. We show that the decay of these miRNAs at the embryo to L1 transition is regulated post-transcriptionally, since mutations in the seed sequence of mir-35 decouple its regulation from that of its clustermates on the same transcript, strongly supporting a regulated decay mechanism.

The seed sequence of mir-35 is not only necessary for this regulated decay, but is also sufficient since mutations in the 3' end of the miRNA do not disrupt decay at the embryo to L1

325 transition. We do observe, however, that the 3' end regulates mir-35 abundance in the embryo, in what appears to be a decay-level effect. We postulate that, whereas a seed-dependent decay 
mechanism enacts developmentally-timed decay, a 3' end-dependent mechanism limits mir-35 abundance in the embryo (Figure S4).

While the TDMD factor ZSWIM8/EBAX-1 regulates mir-35 abundance, our data argues

330 that the mechanisms of mir-35 regulation differ from TDMD in key aspects (Figure 4C-E). First, the decay at embryo to L1 does not require the 3' end sequences which would be involved in base pairing to a typical TDMD trigger RNA. Second, the decay is not accompanied by high levels of tailing or trimming. Furthermore, seed mutations that reduce decay do not reduce tailing or trimming. Together, these data suggest that the mir-35 family is post-transcriptionally regulated

335 by a novel seed-dependent mechanism. We further observed that the altered regulation of seed mutants was not due to loss of target interactions, since restoring these interactions did not restore decay.

We propose a model for mir-35 family decay wherein ZSWIM8/EBAX-1 is recruited to degrade the miRISC in a seed-dependent manner that does not require extensive 3' end base

340 pairing. How the seed is recognized and how ZSWIM8/EBAX-1 is recruited in this process will be an area of ongoing study. Like TDMD, a trigger RNA may base pair with the mir-35 family seed sequence and recruit an RNA binding protein, which can in turn recruit ZSWIM8/EBAX-1 (Figure 4D). Alternatively, the trigger RNA could induce conformational changes in Argonaute that directly recruit ZSWIM8/EBAX-1, similar to proposed models of TDMD. A third possibility is that no trigger

345 RNA is involved in seed recognition for decay; in this case an RBP could bind the mir-35 seed to recruit ZSWIM8/EBAX-1 or induce Argonaute conformational changes (Figure 4E). Because of the large number of possible trigger RNAs or trigger RBPs, further elucidating this mechanism will require large scale biochemical and genetic screens.

Understanding the seed sequence-specific decay mechanism regulating mir-35 will have

350 broad impact. Such seed-specific mechanisms are likely to be present in other biological systems because they allow for simultaneous regulation of redundant paralogs, enabling dynamic regulation of a miRNA seed family's targets. Outside functioning in normal physiology, seedspecific decay mechanisms could be an attractive avenue for modulating abundance of specific miRNA families and their target genes in disease.

\section{Materials and Methods}

General C. elegans culture and maintenance

C. elegans were maintained at $20^{\circ} \mathrm{C}$ on NGM seeded with OP50. For large-scale harvest of 360 embryos, 8,000 starved L1s were plated onto a $10 \mathrm{~cm}$ plate with a large lawn of OP50. The worms 
were re-fed with concentrated OP50 48 hours later. At 96 hours after initial plating, the gravid adults were harvested by bleaching to collect large quantities of embryos.

\section{$\underline{\text { Liquid culture }}$}

For experiments in which deep sequencing was performed (Figures 1 and 2), worms were grown

365 in liquid culture as previously described and harvested with some modifications (Zanin et al., 2011). Briefly, the gravid worms were harvested by centrifugation at $3000 \mathrm{xg}$ for 2 minutes in $50 \mathrm{~mL}$ conical tubes. They were washed once with room temperature water and then pelleted. The volume of the sample was brought up to $28 \mathrm{~mL}$ with water, and then $4 \mathrm{~mL}$ of $5 \mathrm{M} \mathrm{NaOH}$ and $8 \mathrm{~mL}$ of $4 \%$ sodium hypochlorite were added. The tubes were immediately shaken vigorously for 2

370 minutes and allowed to rest on the bench for 1 minute, and this shaking and resting was repeated three times. The worms were immediately centrifuged at $3000 \mathrm{xg}$ for 2 minutes. The supernatant was decanted, and the worms were washed four times with $45 \mathrm{~mL}$ of water. The synchronized embryos were either collected for the embryo samples, or M9 with cholesterol was added, and the worms were placed on a rocker at $20^{\circ} \mathrm{C}$ overnight to obtain a population of synchronized, 375 starved L1 worms.

\section{CRISPR/Cas9-mediated genome editing}

For all CRISPR experiments, pre-assembled Cas9 RNPs were injected into germlines along with short homology-directed repair templates with 35-nt homology arms (Paix et al., 2014). For all CRISPR injections, one of the guide RNAs used targeted $d p y-10$ as a visible marker to select 380 plates with efficient genome editing (Arribere et al., 2014). crRNAs and tracrRNA were ordered from IDT (Alt-R) or Dharmacon (Edit-R), and annealed at $10 \mu \mathrm{M}$ in duplex buffer by heating to $95^{\circ} \mathrm{C}$ for 5 minutes and then cooling to room temperature. Injection mixes contained 2-4 $\mu \mathrm{M}$ Cas9, $4 \mu \mathrm{M}$ total of pre-annealed gRNAs (comprised of gRNAs targeting $d p y-10$ and the site of interest), $0.8 \mu \mathrm{M}$ of the $d p y-10$ donor oligonucleotide, and the homology directed donor at $40-100 \mathrm{ng} / \mu \mathrm{l}$ 385 (Table S2).

Mutations to mir-35 were made by two rounds of CRISPR. First, as previously described (Yang et al., 2020b), two gRNAs recognizing the protospacers TTTCCATTAGAACTATCACC and ATTGCTGGTTTCTTCCACAG were used to create a 50bp deletion at the mir-35 locus. This allele is $\operatorname{mir}-35(c d b 2)$ :

390 GCTGGTTTCTTCCACAGT-50bp_del-CTTTTCCACTTGCTCCAC. The strain carrying mir35(cdb2) was then injected with homology-directed repair donors, along with a gRNA 
(GGAGCAAGTGGAAAAGACTG) recognizing a sequence which is created by the mir-35(cdb2) mutation.

Deep sequencing library preparation and data analysis

395 Library preparation was performed using the NEBNext Small RNA Library Prep Set for Illumina with modifications as previously described (Vieux et al., 2021). Briefly, size selection was performed only after reverse transcription, using $8 \%$ urea gels to purify $\sim 65-75 n$ RT products. Prior to loading on the gel, each RT reaction was treated with 5000units of RNAse H (New England Biolabs) for 30 minutes at $37^{\circ} \mathrm{C}$.

400 Sequence analysis was performed on the NIH High Performance Computing Cluster. The 3' adapter sequence was trimmed using Cutadapt 3.4 (Martin, 2011). The reads were mapped to a custom genome file which was comprised of C. elegans genome WS280 with an additional chromosome containing the sequences of the spike-in miRNAs and the mutant mir-35 precursors with flanking genomic sequence. Mapping was performed using bowtie2 2.4.4 (Langmead et al., 405 2009) with the following settings: --no-unal --end-to-end --sensitive. BAM files were sorted and indexed using samtools 1.13 (Danecek et al., 2021). Reads were assigned to miRNAs using htseq 0.13.5 (Anders et al., 2015) with the following settings: --mode union --nonunique fraction -a 0. The htseq analysis was performed using a gff file modified from mirGeneDB (Fromm et al., 2015) by replacing mirGeneDB IDs with miRbase IDs (Kozomara and Griffiths-Jones, 2014) and adding

410 the intervals corresponding to the spike-in miRNAs and the mir-35 mutant miRNAs in the custom genome file. For analysis of tailing and trimming, the Tailor package (Chou et al., 2015) was used with the genome file described above and FASTA files derived from mirGeneDB, but with IDs replaced by miRbase IDs and sequences for spike-in miRNAs and the mir-35 mutant miRNAs appended.

\section{RNA isolation}

Total RNA was isolated by resuspending the sample in the recommended volume of Trizol reagent (Life Technologies), followed by vortexing at room temperature for fifteen minutes, followed by preparation according to the Trizol manufacturer's instructions.

\section{Taqman miRNA qPCR}

420 For all miRNA qPCR, $5 \mu$ l reverse transcription reactions were performed using the TaqMan MicroRNA Reverse Transcription kit (ThermoFisher). For all samples, 1.66 $\mu$ l of total RNA at $6 \mathrm{ng} / \mu \mathrm{l}$ were used in the reverse transcription. $\mathrm{RT}$ reactions were diluted $1: 4$ and $1.33 \mu \mathrm{l}$ was used in a $5 \mu \mathrm{l}$ qPCR reaction prepared using Taqman miRNA probes with the Taqman Universal 
bioRxiv preprint doi: https://doi.org/10.1101/2021.11.19.469346; this version posted November 20, 2021. The copyright holder for this preprint

(which was not certified by peer review) is the author/funder. This article is a US Government work. It is not subject to copyright under 17 USC 105 and is also made available for use under a CCO license.

Mastermix II with UNG (ThermoFisher). Reactions were run in triplicate on the Applied 425 Biosystems QuantStudio Pro 6.

\section{Data Availability}

All raw sequence data will be deposited in NCBI Sequence Read Archive.

\section{Funding}

This work was funded by the NIDDK Intramural Research Program (ZIA DK075147).

\section{Acknowledgments}

We thank WormBase, the NIDDK Genomics Core, the NCl Genomics Core, and NIH High Performance Computing. Strains are regularly received from the CGC, which is funded by NIH Office of Research Infrastructure Programs (P40 OD010440). Thank you to members of the McJunkin lab, Eric Miska, Kenneth Murfitt, Michael Lichten, Joana Vidigal, John Kim, and Leemor 435 Joshua-Tor for helpful discussions. 


\section{References}

Alvarez-Saavedra, E., and Horvitz, H.R. (2010). Many families of C. elegans microRNAs are not essential for development or viability. Curr. Biol. 20, 367-373.

440 Ameres, S.L., Horwich, M.D., Hung, J.-H., Xu, J., Ghildiyal, M., Weng, Z., and Zamore, P.D. (2010). Target RNA-directed trimming and tailing of small silencing RNAs. Science 328, 15341539.

Anders, S., Pyl, P.T., and Huber, W. (2015). HTSeq-a Python framework to work with highthroughput sequencing data. Bioinformatics 31, 166-169.

445 Arribere, J.A., Bell, R.T., Fu, B.X.H., Artiles, K.L., Hartman, P.S., and Fire, A.Z. (2014). Efficient marker-free recovery of custom genetic modifications with CRISPR/Cas9 in Caenorhabditis elegans. Genetics 198, 837-846.

Baccarini, A., Chauhan, H., Gardner, T.J., Jayaprakash, A.D., Sachidanandam, R., and Brown, B.D. (2011). Kinetic analysis reveals the fate of a microRNA following target regulation in

450 mammalian cells. Curr. Biol. 21, 369-376.

Bail, S., Swerdel, M., Liu, H., Jiao, X., Goff, L.A., Hart, R.P., and Kiledjian, M. (2010). Differential regulation of microRNA stability. RNA 16, 1032-1039.

Bernstein, E., Caudy, A.A., Hammond, S.M., and Hannon, G.J. (2001). Role for a bidentate ribonuclease in the initiation step of RNA interference. Nature 409, 363-366.

455 Bitetti, A., Mallory, A.C., Golini, E., Carrieri, C., Carreño Gutiérrez, H., Perlas, E., Pérez-Rico, Y.A., Tocchini-Valentini, G.P., Enright, A.J., Norton, W.H.J., et al. (2018). MicroRNA degradation by a conserved target RNA regulates animal behavior. Nat. Struct. Mol. Biol. 25, 244-251.

Boele, J., Persson, H., Shin, J.W., Ishizu, Y., Newie, I.S., Sokilde, R., Hawkins, S.M., Coarfa, 460 C., Ikeda, K., Takayama, K. -i., et al. (2014). PAPD5-mediated 3' adenylation and subsequent degradation of miR-21 is disrupted in proliferative disease. Proc. Natl. Acad. Sci. 111, 1146711472.

Bossé, G.D., Rüegger, S., Ow, M.C., Vasquez-Rifo, A., Rondeau, E.L., Ambros, V.R., and Simard, M.J. (2013). The decapping scavenger enzyme DCS-1 controls microRNA levels in

465 Caenorhabditis elegans. Mol. Cell 50, 281-287.

Brancati, G. and Grosshans, H. (2018). An interplay of miRNA abundance and target site architecture determines miRNA activity and specificity. Nucleic Acids Res. 46, 3259-3269

Brennecke, J., Stark, A., Russell, R.B., and Cohen, S.M. (2005). Principles of microRNA-target recognition. PLoS Biol. 3, e85.

470 Broughton, J.P., Lovci, M.T., Huang, J.L., Yeo, G.W., and Pasquinelli, A.E. (2016). Pairing beyond the Seed Supports MicroRNA Targeting Specificity. Mol. Cell 64, 320-333.

Cazalla, D., Yario, T., Steitz, J.A., and Steitz, J. (2010). Down-regulation of a host microRNA by a Herpesvirus saimiri noncoding RNA. Science 328, 1563-1566.

Chatterjee, S., Grosshans, H., and Großhans, H. (2009). Active turnover modulates mature 475 microRNA activity in Caenorhabditis elegans. Nature 461, 546-549.

Chatterjee, S., Fasler, M., Büssing, I., and Grosshans, H. (2011). Target-mediated protection of 
endogenous microRNAs in C. elegans. Dev. Cell 20, 388-396.

Chou, M. Te, Han, B.W., Hsiao, C.P., Zamore, P.D., Weng, Z., and Hung, J.H. (2015). Tailor: a computational framework for detecting non-templated tailing of small silencing RNAs. Nucleic

480 Acids Res. 43.

Dallaire, A., Frédérick, P.-M., and Simard, M.J. (2018). Somatic and Germline MicroRNAs Form Distinct Silencing Complexes to Regulate Their Target mRNAs Differently. Dev. Cell 47, 239247.e4.

Danecek, P., Bonfield, J.K., Liddle, J., Marshall, J., Ohan, V., Pollard, M.O., Whitwham, A., 485 Keane, T., McCarthy, S.A., Davies, R.M., et al. (2021). Twelve years of SAMtools and BCFtools. Gigascience 10.

Denli, A.M., Tops, B.B.J., Plasterk, R.H. a, Ketting, R.F., and Hannon, G.J. (2004). Processing of primary microRNAs by the Microprocessor complex. Nature 432, 231-235.

Dexheimer, P.J., and Cochella, L. (2020). MicroRNAs: From Mechanism to Organism. Front. 490 Cell Dev. Biol. 8, 409.

Dexheimer, P.J., Wang, J., and Cochella, L. (2020). Two microRNAs are sufficient for embryogenesis in C. elegans. BioRxiv 2020.06.28.176024.

Doll, M.A., Soltanmohammadi, N., and Schumacher, B. (2019). ALG-2/AGO-Dependent mir-35 Family Regulates DNA Damage-Induced Apoptosis Through MPK-1/ERK MAPK Signaling

495 Downstream of the Core Apoptotic Machinery in Caenorhabditis elegans. Genetics 213, 173194.

Elbarbary, R.A., Miyoshi, K., Myers, J.R., Du, P., Ashton, J.M., Tian, B., and Maquat, L.E. (2017a). Tudor-SN-mediated endonucleolytic decay of human cell microRNAs promotes G1/S phase transition. Science 356, 859-862.

500 Elbarbary, R.A., Miyoshi, K., Hedaya, O., Myers, J.R., and Maquat, L.E. (2017b). UPF1 helicase promotes TSN-mediated miRNA decay. Genes Dev. 31, 1483-1493.

Fang, W., and Bartel, D.P. (2015). The Menu of Features that Define Primary MicroRNAs and Enable De Novo Design of MicroRNA Genes. Mol. Cell 60, 131-145.

Fromm, B., Billipp, T., Peck, L.E., Johansen, M., Tarver, J.E., King, B.L., Newcomb, J.M.,

505 Sempere, L.F., Flatmark, K., Hovig, E., et al. (2015). A Uniform System for the Annotation of Vertebrate microRNA Genes and the Evolution of the Human microRNAome.

Http://Dx.Doi.Org/10.1146/Annurev-Genet-120213-092023 49, 213-242.

Ghini, F., Rubolino, C., Climent, M., Simeone, I., Marzi, M.J., and Nicassio, F. (2018).

Endogenous transcripts control miRNA levels and activity in mammalian cells by target-directed

510 miRNA degradation. Nat. Commun. 9, 3119.

Gregory, R.I., Yan, K.-P., Amuthan, G., Chendrimada, T., Doratotaj, B., Cooch, N., and Shiekhattar, R. (2004). The Microprocessor complex mediates the genesis of microRNAs. Nature 432, 235-240.

Grishok, A., Pasquinelli, A.E., Conte, D., Li, N., Parrish, S., Ha, I., Baillie, D.L., Fire, A., Ruvkun, 515 G., and Mello, C.C. (2001). Genes and mechanisms related to RNA interference regulate expression of the small temporal RNAs that control C. elegans developmental timing. Cell 106, 23-34.

Grün, D., Kirchner, M., Thierfelder, N., Stoeckius, M., Selbach, M., and Rajewsky, N. (2014). 
Conservation of mRNA and protein expression during development of C. elegans. Cell Rep. 6, 565-577.

Han, J., Lee, Y., Yeom, K.-H., Kim, Y.-K., Jin, H., and Kim, V.N. (2004). The Drosha-DGCR8 complex in primary microRNA processing. Genes Dev. 18, 3016-3027.

Han, J., Lee, Y., Yeom, K.-H., Nam, J.-W., Heo, I., Rhee, J.-K., Sohn, S.Y., Cho, Y., Zhang, B.T., and Kim, V.N. (2006). Molecular basis for the recognition of primary microRNAs by the

525 Drosha-DGCR8 complex. Cell 125, 887-901.

Han, J., LaVigne, C.A., Jones, B.T., Zhang, H., Gillett, F., and Mendell, J.T. (2020). A ubiquitin ligase mediates target-directed microRNA decay independently of tailing and trimming. Science 370 , eabc9546.

Helwak, A., Kudla, G., Dudnakova, T., and Tollervey, D. (2013). Mapping the Human miRNA

530 Interactome by CLASH Reveals Frequent Noncanonical Binding. Cell 153, 654.

Hutvágner, G., McLachlan, J., Pasquinelli, A.E., Bálint, E., Tuschl, T., and Zamore, P.D. (2001). A cellular function for the RNA-interference enzyme Dicer in the maturation of the let-7 small temporal RNA. Science 293, 834-838.

Iwasaki, S., Kobayashi, M., Yoda, M., Sakaguchi, Y., Katsuma, S., Suzuki, T., and Tomari, Y. 535 (2010). Hsc70/Hsp90 chaperone machinery mediates ATP-dependent RISC loading of small RNA duplexes. Mol. Cell 39, 292-299.

Iwasaki, S., Sasaki, H.M., Sakaguchi, Y., Suzuki, T., Tadakuma, H., and Tomari, Y. (2015). Defining fundamental steps in the assembly of the Drosophila RNAi enzyme complex. Nature $521,533-536$.

540 Kagias, K., and Pocock, R. (2015). microRNA regulation of the embryonic hypoxic response in Caenorhabditis elegans. Sci. Rep. 5, 11284.

Kato, M., de Lencastre, A., Pincus, Z., and Slack, F.J. (2009). Dynamic expression of small noncoding RNAs, including novel microRNAs and piRNAs/21U-RNAs, during Caenorhabditis elegans development. Genome Biol. 10, R54.

545 Katoh, T., Hojo, H., and Suzuki, T. (2015). Destabilization of microRNAs in human cells by 3' deadenylation mediated by PARN and CUGBP1. Nucleic Acids Res. 43, 7521-7534.

Ketting, R.F., Fischer, S.E., Bernstein, E., Sijen, T., Hannon, G.J., and Plasterk, R.H. (2001). Dicer functions in RNA interference and in synthesis of small RNA involved in developmental timing in C. elegans. Genes Dev. 15, 2654-2659.

550 Kingston, E.R., and Bartel, D.P. (2019). Global analyses of the dynamics of mammalian microRNA metabolism. Genome Res. 29, 1777-1790.

Kleaveland, B., Shi, C.Y., Stefano, J., and Bartel, D.P. (2018). A Network of Noncoding Regulatory RNAs Acts in the Mammalian Brain. Cell 174, 350-362.e17.

Knight, S.W., and Bass, B.L. (2001). A role for the RNase III enzyme DCR-1 in RNA 555 interference and germ line development in Caenorhabditis elegans. Science 293, 2269-2271.

Knouf, E.C., Wyman, S.K., and Tewari, M. (2013). The human TUT1 nucleotidyl transferase as a global regulator of microRNA abundance. PLoS One 8, e69630.

Kozomara, A., and Griffiths-Jones, S. (2014). MiRBase: Annotating high confidence microRNAs using deep sequencing data. Nucleic Acids Res. 42, D68-D73. 
560 Krol, J., Busskamp, V., Markiewicz, I., Stadler, M.B., Ribi, S., Richter, J., Duebel, J., Bicker, S., Fehling, H.J., Schübeler, D., et al. (2010). Characterizing light-regulated retinal microRNAs reveals rapid turnover as a common property of neuronal microRNAs. Cell 141, 618-631.

Landthaler, M., Yalcin, A., and Tuschl, T. (2004). The Human DiGeorge Syndrome Critical Region Gene 8 and Its D. melanogaster Homolog Are Required for miRNA Biogenesis. Curr.

565 Biol. 14, 2162-2167.

Langmead, B., Trapnell, C., Pop, M., and Salzberg, S.L. (2009). Ultrafast and memory-efficient alignment of short DNA sequences to the human genome. Genome Biol. 10, R25.

Lee, D., Park, D., Park, J.H., Kim, J.H., and Shin, C. (2019). Poly(A)-specific ribonuclease sculpts the $3^{\prime}$ ends of microRNAs. RNA 25, 388-405.

570 Lee, M., Choi, Y., Kim, K., Jin, H., Lim, J., Nguyen, T.A.A., Yang, J., Jeong, M., Giraldez, A.J.J., Yang, H., et al. (2014). Adenylation of maternally inherited microRNAs by Wispy. Mol. Cell 56, 696-707.

Lehrbach, N.J., Castro, C., Murfitt, K.J., Abreu-Goodger, C., Griffin, J.L., and Miska, E.A. (2012). Post-developmental microRNA expression is required for normal physiology, and 575 regulates aging in parallel to insulin/IGF-1 signaling in C. elegans. RNA 18, 2220-2235.

Lewis, B.P., Shih, I., Jones-Rhoades, M.W., Bartel, D.P., and Burge, C.B. (2003). Prediction of mammalian microRNA targets. Cell 115, 787-798.

Libri, V., Helwak, A., Miesen, P., Santhakumar, D., Borger, J.G., Kudla, G., Grey, F., Tollervey, D., and Buck, A.H. (2012). Murine cytomegalovirus encodes a miR-27 inhibitor disguised as a

580 target. Proc. Natl. Acad. Sci. U. S. A. 109, 279-284.

Liu, M., Liu, P., Zhang, L., Cai, Q., Gao, G., Zhang, W., Zhu, Z., Liu, D., and Fan, Q. (2011). mir35 is involved in intestine cell G1/S transition and germ cell proliferation in C. elegans. Cell Res. 21, 1605-1618.

Ma, H., Wu, Y., Choi, J.-G., and Wu, H. (2013). Lower and upper stem-single-stranded RNA 585 junctions together determine the Drosha cleavage site. Proc. Natl. Acad. Sci. U. S. A. 110, 20687-20692.

Marcinowski, L., Tanguy, M., Krmpotic, A., Rädle, B., Lisnić, V.J., Tuddenham, L., ChaneWoon-Ming, B., Ruzsics, Z., Erhard, F., Benkartek, C., et al. (2012). Degradation of cellular mir27 by a novel, highly abundant viral transcript is important for efficient virus replication in vivo.

590 PLoS Pathog. 8, e1002510.

Martin, M. (2011). Cutadapt removes adapter sequences from high-throughput sequencing reads. EMBnet.Journal 17, 10-12.

Marzi, M.J., Ghini, F., Cerruti, B., De Pretis, S., Bonetti, P., Giacomelli, C., Gorski, M.M., Kress, T., Pelizzola, M., Muller, H., et al. (2016). Degradation dynamics of micrornas revealed by a 595 novel pulse-chase approach. Genome Res. 26, 554-565.

Massirer, K.B., Perez, S.G., Mondol, V., and Pasquinelli, A.E. (2012). The miR-35-41 family of microRNAs regulates RNAi sensitivity in Caenorhabditis elegans. PLoS Genet. 8, e1002536.

la Mata, M., Gaidatzis, D., Vitanescu, M., Stadler, M.B., Wentzel, C., Scheiffele, P., Filipowicz, W., and Großhans, H. (2015). Potent degradation of neuronal mi RNA s induced by highly 600 complementary targets . EMBO Rep. 16, 500-511.

McJunkin, K., and Ambros, V. (2014). The embryonic mir-35 family of microRNAs promotes 
multiple aspects of fecundity in Caenorhabditis elegans. G3 (Bethesda). 4, 1747-1754.

McJunkin, K., and Ambros, V. (2017). A microRNA family exerts maternal control on sex determination in C. elegans. Genes Dev. 31, 422-437.

605 Miki, T.S., Rüegger, S., Gaidatzis, D., Stadler, M.B., and Großhans, H. (2014). Engineering of a conditional allele reveals multiple roles of XRN2 in Caenorhabditis elegans development and substrate specificity in microRNA turnover. Nucleic Acids Res. 42, 4056-4067.

Paix, A., Wang, Y., Smith, H.E., Lee, C.Y.S., Calidas, D., Lu, T., Smith, J., Schmidt, H., Krause, M.W., and Seydoux, G. (2014). Scalable and versatile genome editing using linear DNAs with 610 microhomology to Cas9 sites in Caenorhabditis elegans. Genetics 198, 1347-1356.

Parchem, R.J., Moore, N., Fish, J.L., Parchem, J.G., Braga, T.T., Shenoy, A., Oldham, M.C., Rubenstein, J.L.R., Schneider, R.A., and Blelloch, R. (2015). miR-302 Is Required for Timing of Neural Differentiation, Neural Tube Closure, and Embryonic Viability. Cell Rep. 12, 760-773.

Piwecka, M., Glažar, P., Hernandez-Miranda, L.R., Memczak, S., Wolf, S.A., Rybak-Wolf, A.,

615 Filipchyk, A., Klironomos, F., Cerda Jara, C.A., Fenske, P., et al. (2017). Loss of a mammalian circular RNA locus causes miRNA deregulation and affects brain function. Science 357, eaam8526.

Reichholf, B., Herzog, V.A., Fasching, N., Manzenreither, R.A., Sowemimo, I., and Ameres, S.L. (2019). Time-Resolved Small RNA Sequencing Unravels the Molecular Principles of MicroRNA 620 Homeostasis. Mol. Cell 75, 756-768.e7.

Rissland, O.S., Hong, S.-J., and Bartel, D.P. (2011). MicroRNA destabilization enables dynamic regulation of the miR-16 family in response to cell-cycle changes. Mol. Cell 43, 993-1004.

Sherrard, R., Luehr, S., Holzkamp, H., McJunkin, K., Memar, N., and Conradt, B. (2017). Mirnas cooperate in apoptosis regulation during c. Elegans development. Genes Dev. 31, 209-222.

625 Sheu-Gruttadauria, J., Pawlica, P., Klum, S.M., Wang, S., Yario, T.A., Schirle Oakdale, N.T., Steitz, J.A., and MacRae, I.J. (2019). Structural Basis for Target-Directed MicroRNA Degradation. Mol. Cell 75, 1243-1255.e7.

Shi, C.Y., Kingston, E.R., Kleaveland, B., Lin, D.H., Stubna, M.W., and Bartel, D.P. (2020). The ZSWIM8 ubiquitin ligase mediates target-directed microRNA degradation. Science 370,

630 eabc9359.

Shukla, S., Bjerke, G.A., Muhlrad, D., Yi, R., and Parker, R. (2019). The RNase PARN Controls the Levels of Specific miRNAs that Contribute to p53 Regulation. Mol. Cell 73, 1204-1216.e4.

Stoeckius, M., Maaskola, J., Colombo, T., Rahn, H.-P., Friedländer, M.R., Li, N., Chen, W., Piano, F., and Rajewsky, N. (2009). Large-scale sorting of C. elegans embryos reveals the 635 dynamics of small RNA expression. Nat. Methods 6, 745-751.

Tran, A.T., Chapman, E.M., Flamand, M.N., Yu, B., Krempel, S.J., Duchaine, T.F., Eroglu, M., and Derry, W.B. (2019). MiR-35 buffers apoptosis thresholds in the C. elegans germline by antagonizing both MAPK and core apoptosis pathways. Cell Death Differ. 26, 2637-2651.

Vieux, K.-F., Prothro, K.P., Kelley, L.H., Palmer, C., Maine, E.M., Veksler-Lublinsky, I., and 640 McJunkin, K. (2021). Screening by deep sequencing reveals mediators of microRNA tailing in C. elegans. Nucleic Acids Res. 49, 11167-11180.

Wang, Z., Hou, Y., Guo, X., vanderVoet, M., Boxem, M., Dixon, J.E., Chisholm, A.D., and Jin, Y. (2013). The EBAX-type Cullin-RING E3 Ligase and Hsp90 Guard the Protein Quality of the 
SAX-3/Robo Receptor in Developing Neurons. Neuron 79, 903-916.

645 Wu, E., Thivierge, C., Flamand, M., Mathonnet, G., Vashisht, A.A., Wohlschlegel, J., Fabian, M.R., Sonenberg, N., and Duchaine, T.F. (2010). Pervasive and cooperative deadenylation of 3'UTRs by embryonic microRNA families. Mol. Cell 40, 558-570.

Wyman, S.K., Knouf, E.C., Parkin, R.K., Fritz, B.R., Lin, D.W., Dennis, L.M., Krouse, M.A., Webster, P.J., and Tewari, M. (2011). Post-transcriptional generation of miRNA variants by 650 multiple nucleotidyl transferases contributes to miRNA transcriptome complexity. Genome Res. $21,1450-1461$.

Yang, A., Shao, T.-J., Bofill-De Ros, X., Lian, C., Villanueva, P., Dai, L., and Gu, S. (2020a). AGO-bound mature miRNAs are oligouridylated by TUTs and subsequently degraded by DIS3L2. Nat. Commun. 11, 2765.

655 Yang, B., Schwartz, M., and McJunkin, K. (2020b). In vivo CRISPR screening for phenotypic targets of the mir-35-42 family in C. elegans. Genes Dev. 34, 1227-1238.

Ye Duan, Isana Veksler-Lublinsky, V.A. (2021). Critical contribution of 3' non-seed base pairing to the in vivo function of the evolutionarily conserved let-7a microRNA. Biorxiv.

Zanin, E., Dumont, J., Gassmann, R., Cheeseman, I., Maddox, P., Bahmanyar, S., Carvalho, A., 660 Niessen, S., Yates, J.R., Oegema, K., et al. (2011). Affinity purification of protein complexes in C. elegans. Methods Cell Biol. 106, 289-322.

Zeng, Y., Yi, R., and Cullen, B.R. (2005). Recognition and cleavage of primary microRNA precursors by the nuclear processing enzyme Drosha. EMBO J. 24, 138-148.

Zhao, Y., Jin, L., Wang, Y., Kong, Y., and Wang, D. (2019). Prolonged exposure to multi-walled 665 carbon nanotubes dysregulates intestinal mir-35 and its direct target MAB-3 in nematode Caenorhabditis elegans. Sci. Rep. 9. 
A

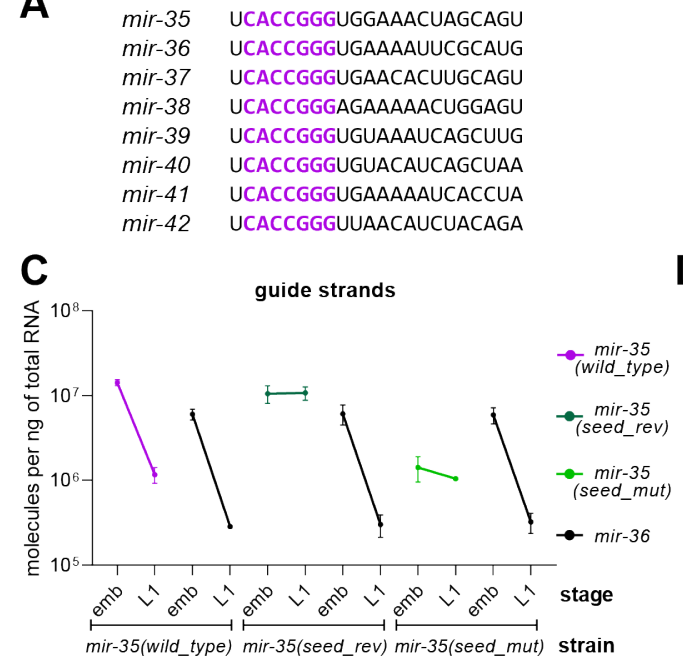

E
B

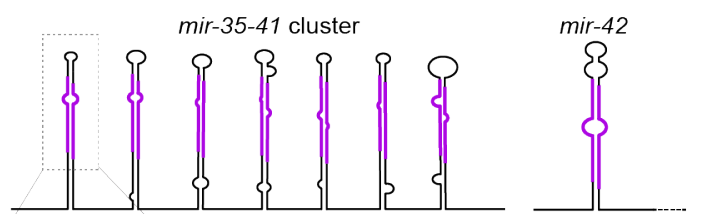

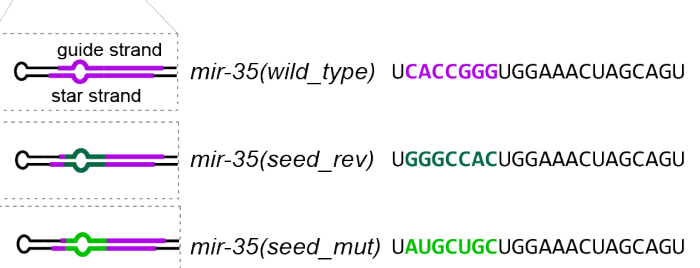
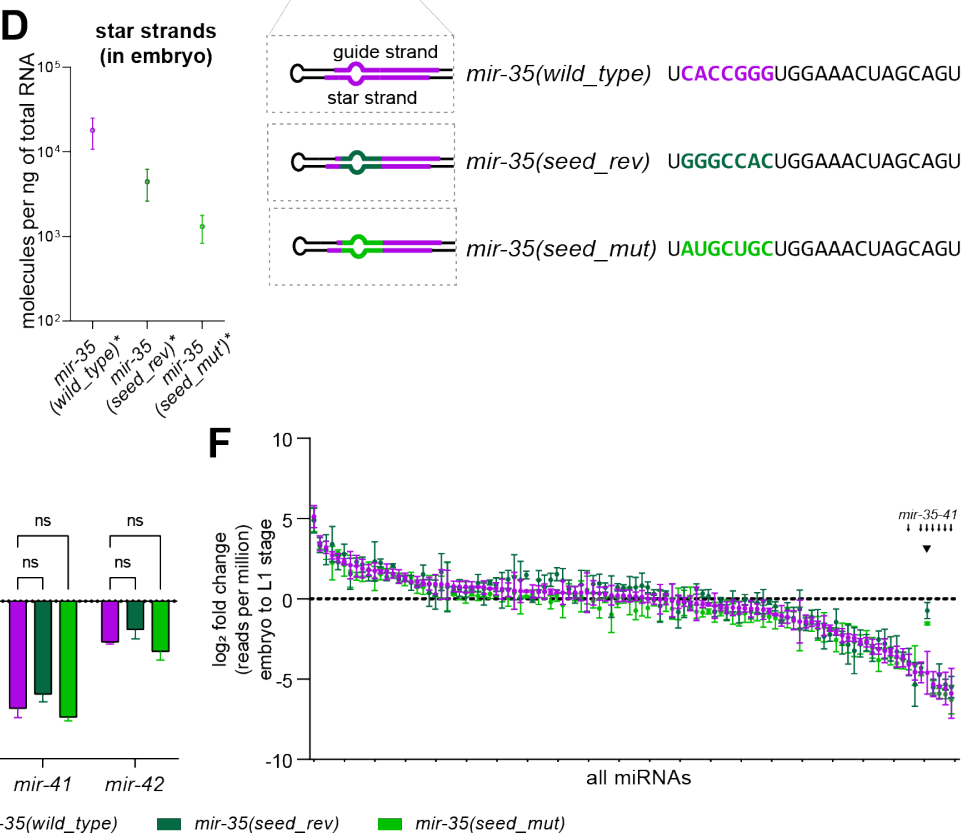

670 Figure 1. mir-35 decay is seed sequence-dependent (A) Sequences of mirs-35-42. The identical seed sequence is shown in purple. (B) Schematic of the mir-35-41 cluster and mir-42 showing the structure of the hairpins in the primary transcripts. The guide and star strands are highlighted in purple (top). Below is the wild type mir-35 sequence (purple) and the two seed mutant mir-35 variants (green). Both seed mutant mir-35 variants have compensatory mutations 675 to the star strand to maintain the wild type-like structure of the mutant mir-35 hairpin. (C) Absolute quantification of mir-35 and mir-36 in embryos and L1. For some error bars, the range is smaller than the symbol. (D) Absolute quantification of star strands of mir-35 and mutant variants in embryos. (E-F) $\log _{2}$ (fold change) in abundance from embryo to $L 1$, calculated from normalized deep sequencing reads for either the mir-35-42 family (E) or all miRNAs with $>50$ RPM in wild type $(F)$. Note that color of bar indicates strain, not necessarily a mutant miRNA; only mir-35 is mutated in the indicated mutant strains. (E) Two-way ANOVA was performed, followed by Dunnett's multiple comparisons test. ${ }^{* * * *}$ p-value $<0.0001$ Small arrows in $(F)$ indicate positions of mir-35-41 on ranked X-axis, and arrowhead indicates mir-35 with mutant variants. (C-F) Mean and SEM of three biological replicates are shown. 
A

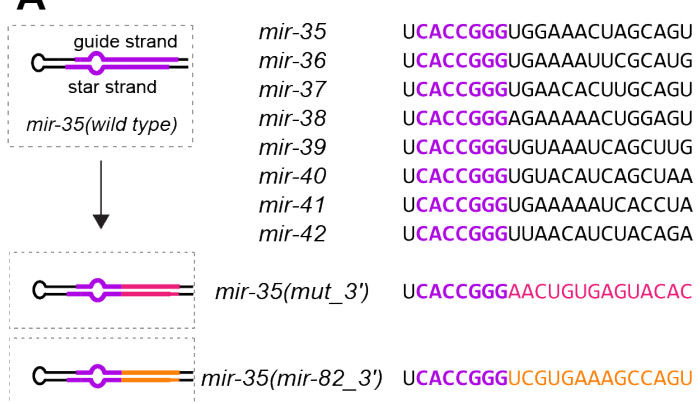

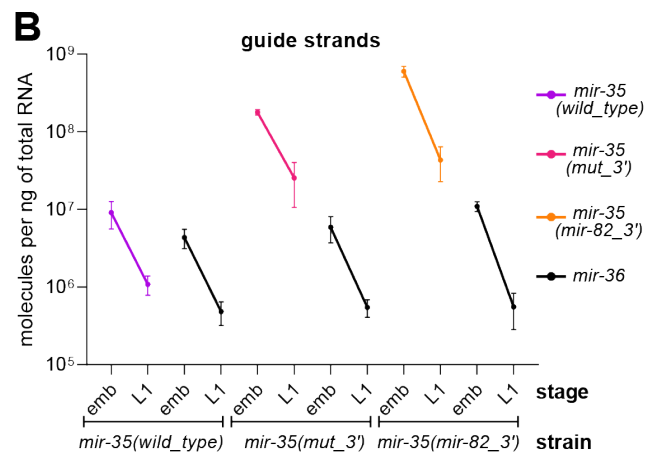

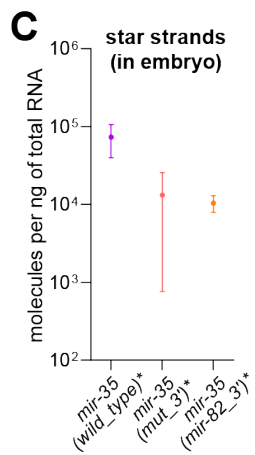

D

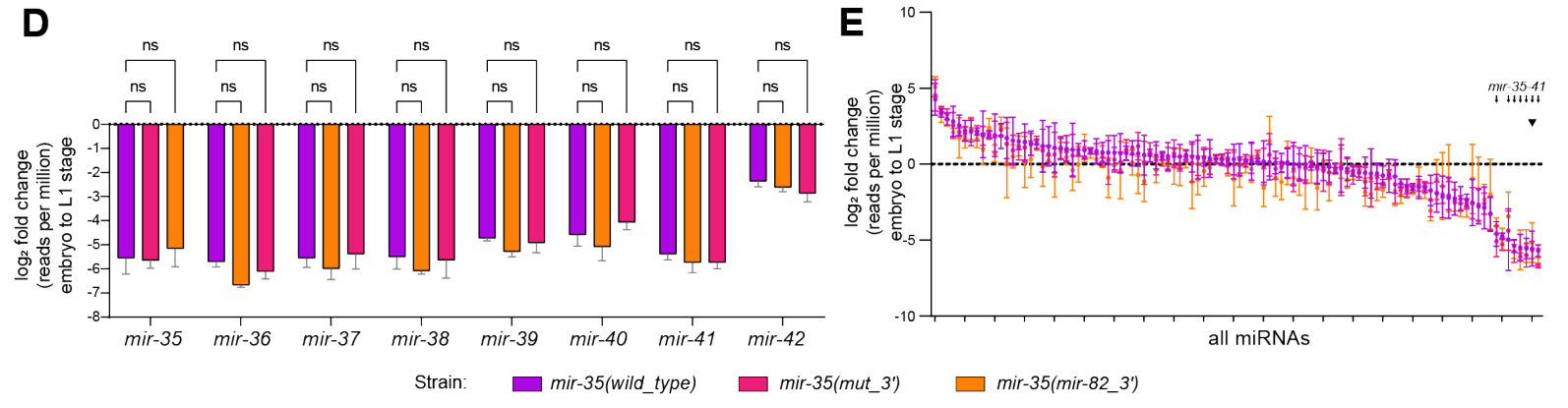

Figure 2. mir-35 3' end mutants do not alter decay (A) Sequences of mirs-35-42 with the identical seed sequences shown in purple (top). Schematic of the mir-35 3' end mutants (bottom). Both 3' end mutants have compensatory mutations to the star strand to maintain the wild type like structure of the mutant mir-35 hairpin. (B) Absolute quantification of mir-35 and mir-36 in embryos and L1. Mean and SEM of two to three biological replicates are shown. (C) Absolute quantification of star strands of mir-35 and mutant variants in embryos. (D-E) $\log _{2}$ (fold change) in abundance from embryo to $L 1$, calculated from normalized deep sequencing reads for either the mir-35-42 family (D) or all miRNAs with $>50$ RPM in wild type (E). Note that color of bar indicates strain, not necessarily a mutant miRNA; only mir-35 is mutated in the indicated mutant strains. (D) Two-way ANOVA was performed, followed by Dunnett's multiple comparisons test. Small arrows in (E) indicate positions of mir-35-41 on ranked x-axis, and arrowhead indicates mir-35 with mutant variants. (C-E) Mean and SEM of three biological replicates are shown. 

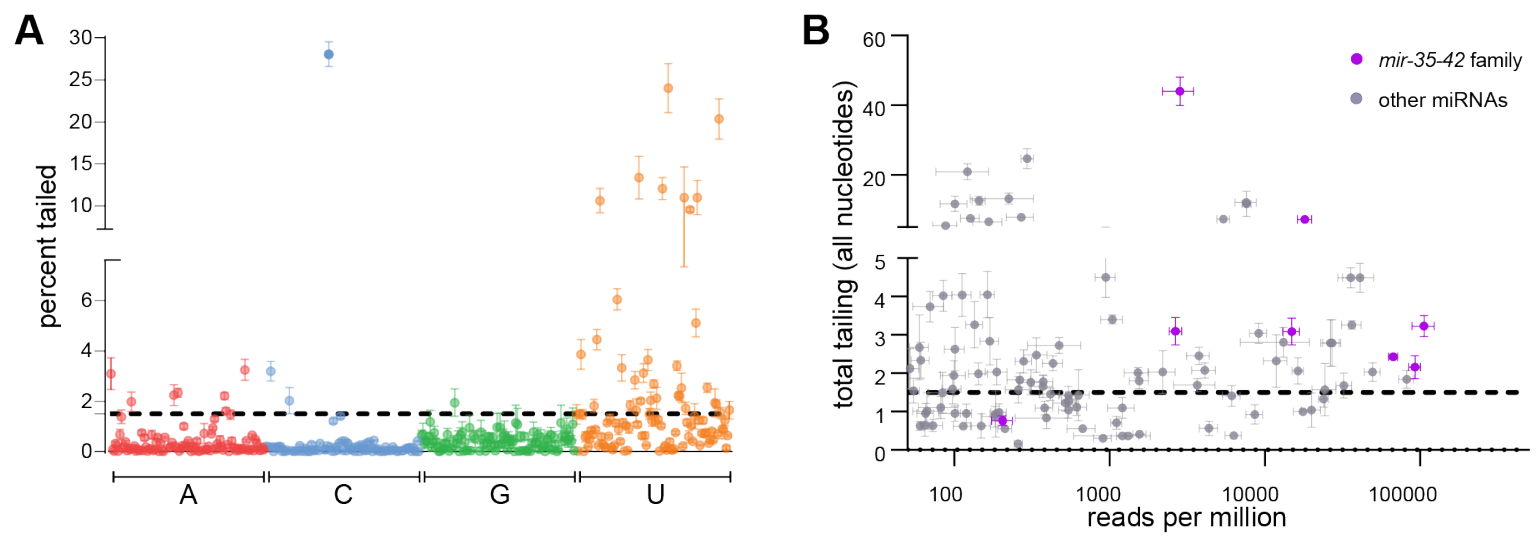

$\mathbf{C}$

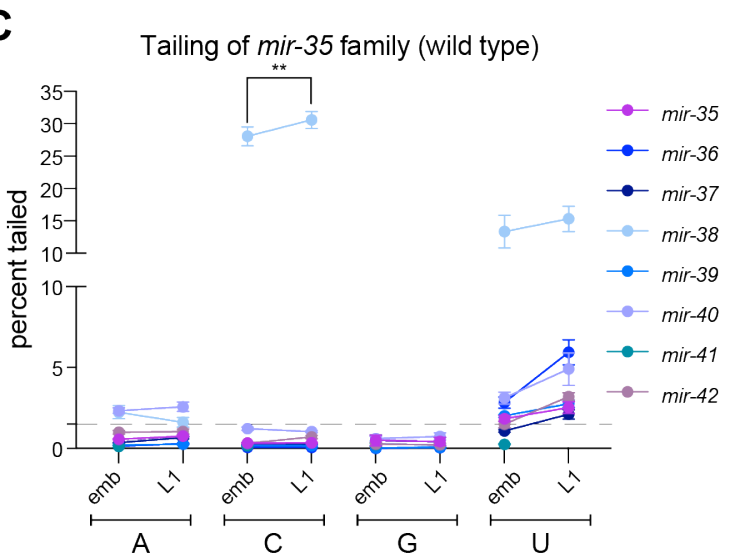

D

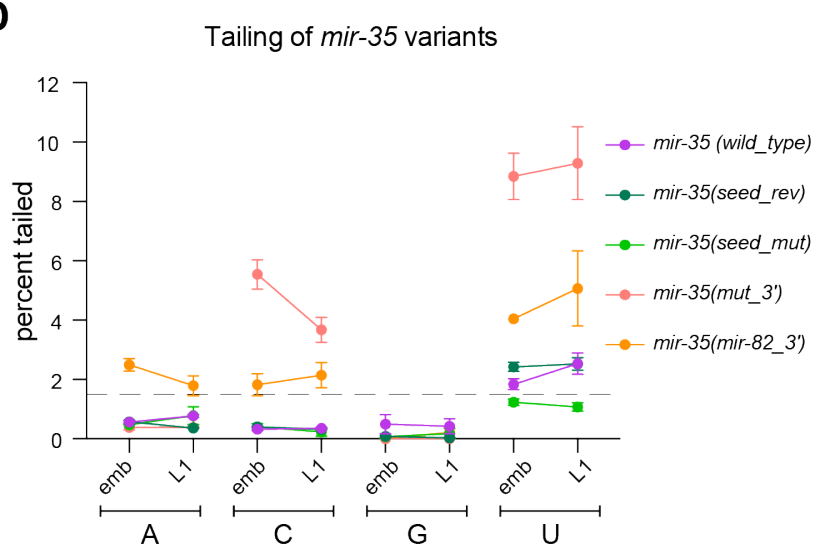

E

Trimming of mir-35 (wild type)

$\mathbf{F}$
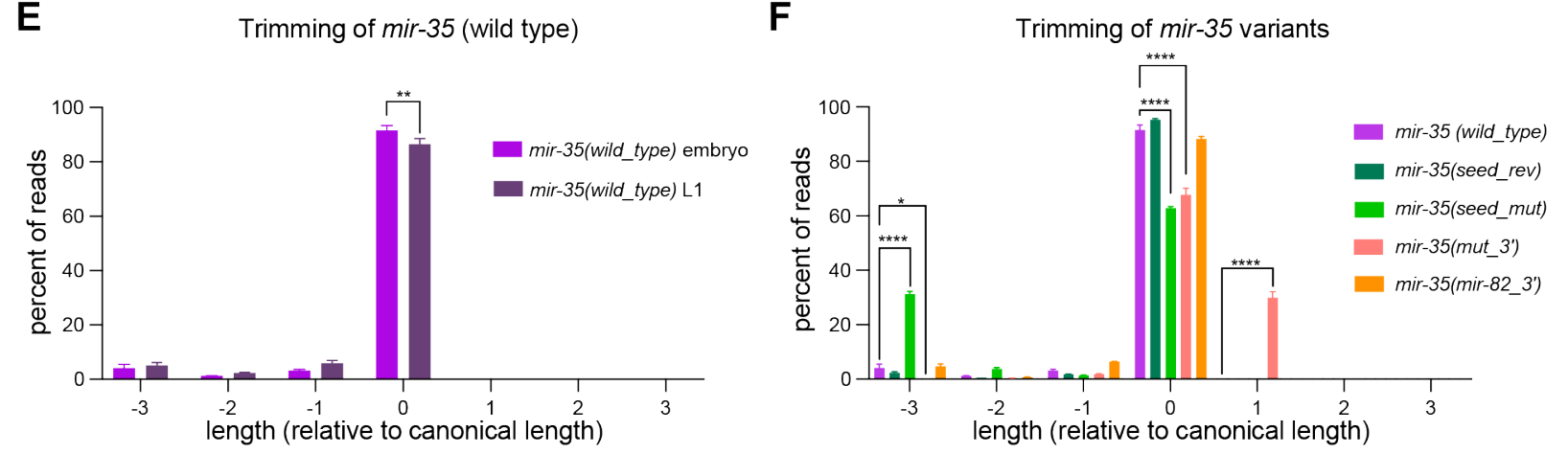

Figure 3. Changes in tailing and trimming of mir-35 variants do not correlate with changes in decay $(A)$ Percent of reads with single nucleotide addition to the 3 ' end is shown for each 705 miRNA with $>50$ RPM in embryo. (B) Total tailing (sum of all single nucleotide tails) versus abundance (RPM) is shown for all miRNAs with $>50$ RPM in embryo. The mir-35 family is highlighted in purple. (C-D) Percent of reads with single nucleotide additions to the mir-35 family (C) and the mir-35 variants (D) in the embryo and L1. (E-F) Percent of reads of each length (excluding tail), relative to the canonical length of mir-35 in wild type embryo and L1 (E) or mir-35 710 variants in embryo (F). (A-F) Mean and SEM are shown for six biological replicates for wild type and three biological replicates for all mir-35 variant strains. (C-F) For each nucleotide, one-way ANOVA was performed, followed by Sidak's multiple comparison test. Significant relationships in panel $D$ are described in the text, but not indicated on the graph for simplicity. ${ }^{*} p$-value $<0.05$, ${ }^{* *} p$-value $<0.01,{ }^{* * * *} p$-value $<0.0001$ 
A
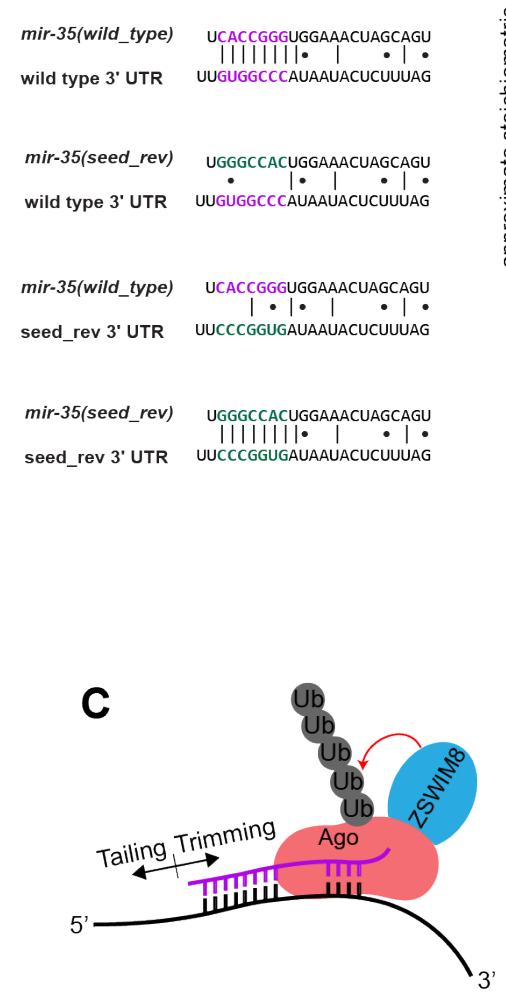

\section{B}
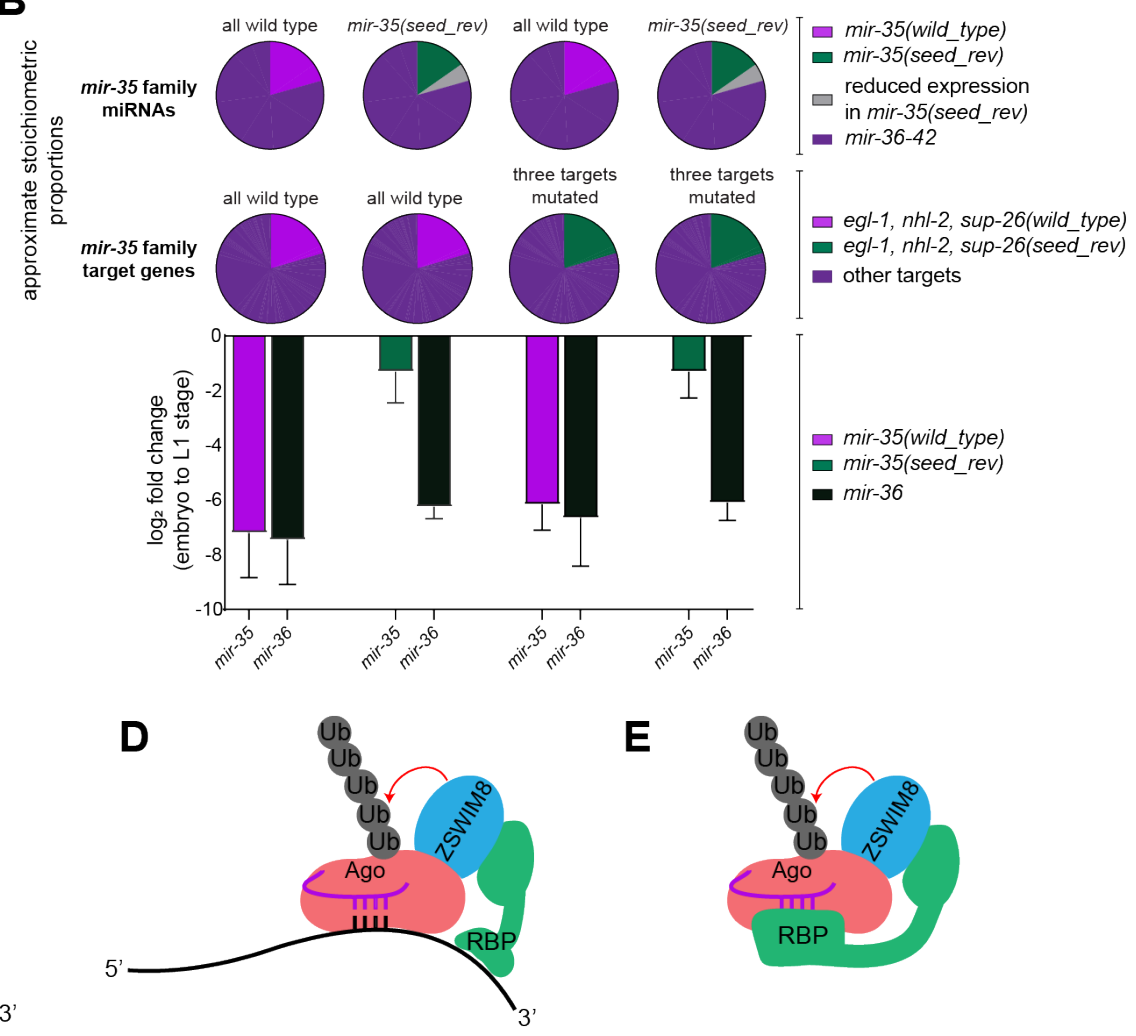

720 Figure 4. Reintroducing miRNA-target interactions does not restore decay of a seed mutant variant of mir-35 (A) Target binding sites for mir-35(seed_rev) were introduced to the 3'UTR of three known mir-35 targets (egl-1, sup-26 and nhl-2), either in a mir-35(wild_type) or a mir35(seed_rev) background. An example of the relevant miRNA-target interactions at the egl-1 3'UTR are shown. (B) Top: Pie charts represent an estimate of the proportion of the mir-35 family 725 molecules and the mir-35 family target molecules that are mutated in each strain. Bottom: $\mathrm{Log}_{2}$ (fold change) from embryo to L1 in the indicated strains, as measured by Taqman qPCR. Mean and SEM of three biological replicates are shown. (C) Model of conventional TDMD. (D-E) Alternative models for regulation of mir-35 family decay, in which the seed sequence is recognized by a complementary RNA (D) or an RNA-binding protein (RBP) (E). 